\title{
Assessment of differences on inflammatory and metabolic indicators between pre- and post-menopause women among hypertensive and/or diabetic patients
}

\begin{abstract}
To assess the differences on inflammatory and metabolic indicators between pre-menopause and post-menopause women among hypertensive and/or diabetic type- 2 women. A total of 236 obese women included in the study have chosen from Primary Health Care Centers in Gaza City, Palestine, through a cross-sectional study. Selection depended on health status hypertensive and/or diabetic type-2 (HT, T2DM, HT+T2DM). In HT group, post-menopause women had significant higher values than pre-menopause women on TC $(200 \pm 47$ vs. $172.5 \pm 55 \mathrm{mg} \mathrm{dL}-1, \mathrm{p}<0.01)$ and TG $(166 \pm 89$ vs. $120.5 \pm 82 \mathrm{mg} \mathrm{dL}-1, \mathrm{p}<0.01)$. In T2DM group, post-menopause women had significant higher values than pre-menopause women on SBP $(132 \pm 24$ vs. $120 \pm 20 \mathrm{~mm} \mathrm{Hg}, \mathrm{p}<0.01)$, TC $(213 \pm 40$ vs. $185 \pm 46 \mathrm{mg} \mathrm{dL}-1, \mathrm{p}<0.05)$ and TG (196 \pm 118 vs. $136 \pm 64 \mathrm{mg} \mathrm{dL}-1, \mathrm{p}<0.05)$. Finally, in HT+T2DM group, post-menopause women had significant higher value than pre-menopause women on SBP (144 \pm 21 vs. $133 \pm 14$ $\mathrm{mmHg}, \mathrm{p}<0.05)$, TC $(214 \pm 54$ vs. $181 \pm 55 \mathrm{mg} \mathrm{dL}-1, \mathrm{p}<0.05)$, TG $(231 \pm 83$ vs. $158 \pm 85 \mathrm{mg} \mathrm{dL}-$ $1, \mathrm{p}<0.05)$, IL-6 (2.32 \pm 1.34 vs. $1.71 \pm 1.45 \mathrm{pg} \mathrm{mL}-1, \mathrm{p}<0.05)$ and BMI $(36.48 \pm 7.1$ vs. $\left.32.18 \pm 5.6 \mathrm{~kg} \mathrm{~m}^{-} 2, \mathrm{p}<0.05\right)$. Post-menopause women diseased of HT and T2DM accompanied with higher rates of BMI are at risk for developing inflammatory and metabolic morbidities.
\end{abstract}

